\title{
A General Way of Constructing Symmetric Gabor Frames
}

\author{
Guochang $\mathrm{Wu}^{1, \mathrm{a}^{*}}$ and Xiuqin Yang ${ }^{2, \mathrm{~b}}$ \\ ${ }^{1}$ School of Arts and Science, Sias International College of Zhengzhou University, \\ Xinzheng 451150, China \\ ${ }^{2}$ Department of Mathematics, Jiaozuo Teachers College, Jiaozuo 454000, China \\ ajinzhouli1962@163.com, ${ }^{b}$ xiuqinyang_hd@163.com
}

Keywords: Signal processing; Gabor system; Frame; Symmetric property

\begin{abstract}
Gabor frames with redundancy property can achieve efficient reconstruction, which play a vital role in applied mathematics and engineering fields. In this paper, symmetric or antisymmetric Gabor frames about any symmetric points with any invertible matrices in higher dimensions are constructed from Gabor frames given. The existing results with symmetric property about origin in one dimension are generalized to the case of any invertible matrices and any symmetric points in higher dimensions.
\end{abstract}

\section{Introduction}

Frames were first found and introduced by Duffin and Schaeffer [1] who studied nonharmonic Fourier series. Then, the important work of Daubechies, Grossmann, and Meyer [2] attracted many people's attention. Since then, the theory of frames began to be more widely studied. Traditionally, frames have been used in signal processing, image processing, data compression and sampling theory. Recently, frames are also used to other engineering fields such as quantization[3] and coding theory and data transmission technology [4].

The first Gabor system was defined and studied by Gabor [5] in 1946, with the Gaussian function in order to construct efficient, time-frequency localized expansions of finite energy signals as (infinite) linear combinations of elements. They are generated by modulations and translations of some fixed functions. That is, by choosing some fixed functions $g^{m} \subset L^{2}\left(R^{n}\right), m=1,2, \cdots, M$ and two invertible matrices $A, B$, we can define the Gabor system $G(g, A, B)$ by

$$
G(g, A, B)=\left\{E_{A l} T_{B k} g^{m}: k \in Z^{n}, \quad l \in Z^{n}, m=1,2, \cdots, M\right\},
$$

where $T_{A k} f(x)=f(x-A k)$ and $E_{B l} f(x)=e^{2 \pi B l x} f(x)$.

An important development about Gabor systems is due to Daubechies, Grossmann and Meyer [2] who placed the problem of Gabor expansions in the framework of frames for a Hilbert space and studied them.

Gabor analysis in real straight line has been widely studied by many people from 1970 and main fundamental and important questions have been successfully solved by means of harmonic analysis and complex analysis, and so on. The main characterization about Gabor frames is provided due to works of [6] and [7]. Authors [8] gave the sufficient conditions in time domain for the Gabor system to be a frame. Then, the monograph of Grochenig [9] discussed in detail time frequency analysis and Gabor systems.

The symmetry plays an important role in engineering because it can reduce image's distortion. In [10], authors gave a simple way to construct symmetric or antisymmetric wavelet frames from any wavelet frames given. Motivated by paper [11], some authors discussed the case of Gabor system [11]. Symmetric or antisymmetric Gabor frames with several generators in integer sampling lattices are constructed. At last, some examples are provided to prove the theory.

In this paper, some results of [11] with symmetric property about origin in one dimension are generalized to the case of any invertible matrices and any symmetric points in higher dimensions. Symmetric or antisymmetric Gabor frames about any symmetric points with any invertible matrices in 
higher dimensions are constructed from Gabor frames given. In special, symmetric or antisymmetric Parseval Gabor frames are obtained. We borrow some thoughts of existing result in wavelet analysis and Gabor analysis. At last, some symmetric or antisymmetric examples are given to prove our theory.

\section{Preliminaries}

In this paper, $R^{n}$ and $Z^{n}$ denote real number set and the integer set in the $\mathrm{n}$-dimensional, respectively. $L^{2}\left(R^{n}\right)$ is the space of all square-integrable functions of the n-dimensional, and $\langle\cdot, \cdot\rangle$ denotes the inner product in $L^{2}\left(R^{n}\right)$.

The definition of frame is given as the following.

Definition 2.1. Let $H$ be a separable Hilbert space. A sequence $\left\{f_{i}\right\}_{i \in N}$ of elements of $H$ is a frame for $H$ if there exist constants $0<C \leq D<\infty$ such that for all $f \in H$, we have

$$
C\|f\|^{2} \leq \sum_{i \in N}\left\|\left\langle f, f_{i}\right\rangle\right\|^{2} \leq D\|f\|^{2} \text {. }
$$

The numbers $C, D$ are called lower and upper frame bounds, respectively (the largest $C$ and the smallest $D$ for which (2) holds are the optimal frame bounds). Those sequences which satisfy only the upper inequality in (2) are called Bessel sequences. A frame is tight if $C=D$. If $C=D=1$, it is called a Parseval frame.

Let $T_{f}$ denote the synthesis operator of $f=\left\{f_{i}\right\}_{i \in N}$, i.e., $T_{f}(c)=\sum_{i} c_{i} f_{i}$ for each sequence of scalars $c=\left(c_{i}\right)_{i \in N}$. Then the frame operator $S h=T_{f} T_{f}^{*}(h)$ associated with $\left\{f_{i}\right\}_{i \in N}$ is a bounded, invertible, and positive operator mapping of $H$ on itself. This provides the reconstruction formula

$$
h=\sum_{i=1}^{\infty}<h, \tilde{f}_{i}>f_{i}=\sum_{i=1}^{\infty}<h, f_{i}>\tilde{f}_{i}, \forall h \in H .
$$

where $\tilde{f}_{i}=S^{-1} f_{i}$. The family $\left\{\tilde{f}_{i}\right\}_{i \in N}$ is also a frame for $H$ and is called the canonical dual frame of $\left\{f_{i}\right\}_{i \in N}$. If $\left\{g_{i}\right\}_{i \in N}$ is any sequence in $H$ which satisfies

$$
h=\sum_{i=1}^{\infty}<h, g_{i}>f_{i}=\sum_{i=1}^{\infty}<h, f_{i}>g_{i}, \forall h \in H,
$$

it is called an alternate dual frame of $\left\{f_{i}\right\}_{i \in N}$.

In the following, we give the definitions of Gabor frame.

Definition 2.2. We say that the Gabor system defined by Eq. 1 is a Gabor frame if it is a frame for $L^{2}\left(R^{n}\right)$.

\section{Main Results}

In this section, we will consider constructing symmetric Gabor frames with any invertible matrices and with any symmetric points in higher dimensions from any Gabor frames given. We generalize the existing result in one dimension [11] to the case of any invertible matrices and any symmetric points in higher dimensions.

For fixed functions $g^{m} \subset L^{2}\left(R^{n}\right), m=1,2, \cdots, M$ and $y \in R^{n}$, define new symmetric or antisymmetric functions about any symmetric points $y$ as the following: 


$$
\begin{aligned}
& g_{1}{ }^{m}(x)=\frac{g^{m}(y+x)+g^{m}(y-x)}{2}, \\
& g_{2}{ }^{m}(x)=\frac{g^{m}(y+x)-g^{m}(y-x)}{2} .
\end{aligned}
$$

Thus, we have

Theorem 3. Let $A, B$ be any invertible matrices. Suppose that Gabor system $\left\{E_{A l} T_{B k} g^{m}: k \in Z^{n}, l \in Z^{n}, m=1,2, \cdots, M\right\}$

defined by (1) is a frame for $L^{2}\left(R^{n}\right)$ with frame bounds $C_{1}, C_{2}$, then Gabor system

$$
\left\{E_{A l} T_{B k} g_{1}{ }^{m} \cup E_{A l} T_{B k} g_{2}{ }^{m}: k \in Z^{n}, \quad l \in Z^{n}, m=1,2, \cdots, M\right\}
$$

is a symmetric or antisymmetric frame for $L^{2}\left(R^{n}\right)$ about any symmetric points $y$ with frame bounds $C_{1}, C_{2}$, where the functions $g_{1}{ }^{m}(x), g_{2}{ }^{m}(x)$ are defined by Eq. 5 .

Proof. Since Gabor system

$$
\left\{E_{A l} T_{B k} g^{m}: k \in Z^{n}, l \in Z^{n}, m=1,2, \cdots, M\right\}
$$

defined by (1) is a frame with frame bounds $C_{1}, C_{2}$, for all $L^{2}\left(R^{n}\right)$, we can get

$$
C_{1}\|f\|^{2} \leq\left.\sum_{m=1}^{M} \sum_{l \in Z^{n}} \sum_{k \in Z^{n}}\left\langle f, E_{A l} T_{B k} g^{m}\right\rangle\right|^{2} \leq C_{2}\|f\|^{2} .
$$

In order to prove above main result, we will calculate the series

$$
\left.\sum_{m=1}^{M} \sum_{l \in Z^{n}} \sum_{k \in Z^{n}}\left\langle f, E_{A l} T_{B k} g_{1}{ }^{m}\right\rangle\right|^{2}+\left.\sum_{m=1}^{M} \sum_{l \in Z^{n}} \sum_{k \in Z^{n}}\left\langle f, E_{A l} T_{B k} g_{2}{ }^{m}\right\rangle\right|^{2} .
$$

From definition of $g_{1}{ }^{m}$ and the property of inner product, we can easily deduce

$$
\left|\left\langle f(\cdot), E_{A l} T_{B k} g_{1}{ }^{m}\right\rangle\right|^{2}=\left|\left\langle f(\cdot), E_{A l} T_{B k} \frac{g^{m}(y+\cdot)}{2}\right\rangle+\left\langle f(\cdot), E_{A l} T_{B k} \frac{g^{m}(y-\cdot)}{2}\right\rangle\right|^{2}
$$

When $z_{1}, z_{2}$ are any complex numbers, we have

$$
\left|z_{1}+z_{2}\right|^{2}=\left|z_{1}\right|^{2}+\left|z_{2}\right|^{2}+z_{1} \overline{z_{2}}+\overline{z_{1}} z_{2} .
$$

According to Eq. 9 and Eq. 10, we have

$$
\begin{aligned}
& \left.\left\langle f(\cdot), E_{A l} T_{B k} g_{1}^{m}(\cdot)\right\rangle\right|^{2}=\frac{1}{4}\left|\left\langle f(\cdot), E_{A l} T_{B k} g^{m}(y+\cdot)\right\rangle\right|^{2}+\frac{1}{4}\left|\left\langle f(\cdot), E_{A l} T_{B k} g^{m}(y-\cdot)\right\rangle\right|^{2} \\
& +\frac{1}{4}\left\langle f(\cdot), E_{A l} T_{B k} g^{m}(y+\cdot)\right\rangle \overline{\left\langle f(\cdot), E_{A l} T_{B k} g^{m}(y-\cdot)\right\rangle} \\
& +\frac{1}{4} \overline{\left\langle f(\cdot), E_{a l} T_{b k} g^{m}(y+\cdot)\right\rangle}\left\langle f(\cdot), E_{a l} T_{b k} g^{m}(y-\cdot)\right\rangle .
\end{aligned}
$$

Similar to Eq. 11, we get

$$
\begin{aligned}
& \left.\left\langle f(\cdot), E_{A l} T_{B k} g_{1}{ }^{m}(\cdot)\right\rangle\right|^{2}=\frac{1}{4}\left|\left\langle f(\cdot), E_{A l} T_{B k} g^{m}(y+\cdot)\right\rangle\right|^{2}+\frac{1}{4}\left|\left\langle f(\cdot), E_{A l} T_{B k} g^{m}(y-\cdot)\right\rangle\right|^{2} \\
& -\frac{1}{4}\left\langle f(\cdot), E_{A l} T_{B k} g^{m}(y+\cdot)\right\rangle \overline{\left\langle f(\cdot), E_{A l} T_{B k} g^{m}(y-\cdot)\right\rangle} \\
& -\frac{1}{4} \overline{\left\langle f(\cdot), E_{a l} T_{b k} g^{m}(y+\cdot)\right\rangle}\left\langle f(\cdot), E_{a l} T_{b k} g^{m}(y-\cdot)\right\rangle .
\end{aligned}
$$


From Eq. 11 and Eq. 12, we have

$$
\begin{aligned}
& \left.\sum_{m=1}^{M} \sum_{l \in Z^{n}} \sum_{k \in Z^{n}}\left\langle f, E_{A l} T_{B k} g_{1}{ }^{m}\right\rangle\right|^{2}+\left.\sum_{m=1}^{M} \sum_{l \in Z^{n}} \sum_{k \in Z^{n}}\left\langle f, E_{A l} T_{B k} g_{2}{ }^{m}\right\rangle\right|^{2} \\
& =\frac{1}{2} \sum_{m=1}^{M} \sum_{l \in Z} \sum_{k \in Z}\left|\left\langle f(\cdot), E_{a l} T_{b k} g^{m}(y+\cdot)\right\rangle\right|^{2} \\
& +\frac{1}{2} \sum_{m=1}^{M} \sum_{l \in Z^{n}} \sum_{k \in Z^{n}}\left|\left\langle f(\cdot), E_{A l} T_{B k} g(y-\cdot)\right\rangle\right|^{2} .
\end{aligned}
$$

Therefore, we easily get

$$
\sum_{m=1}^{M} \sum_{l \in Z^{n}} \sum_{k \in Z^{n}}\left|\left\langle f(\cdot), E_{A l} T_{B k} g^{m}(y-\cdot)\right\rangle\right|^{2}=\sum_{m=1}^{M} \sum_{l \in Z^{n}} \sum_{k \in Z^{n}}\left|\left\langle f(y-\cdot), E_{A l} T_{B k} g^{m}(\cdot)\right\rangle\right|^{2} .
$$

By Eq. 7, we have

$$
C_{1}\|f(y-\cdot)\|^{2} \leq\left.\sum_{m=1}^{M} \sum_{l \in Z^{n}} \sum_{k \in Z^{n}}\left\langle f(y-\cdot), E_{A l} T_{B k} g^{m}\right\rangle\right|^{2} \leq C_{2}\|f(y-\cdot)\|^{2} .
$$

According to Eq. 14 and 15 , the equality $\|f(y-\cdot)\|^{2}=\|f(\cdot)\|^{2}$, we deduce

$$
C_{1}\|f\|^{2} \leq\left.\sum_{m=1}^{M} \sum_{l \in Z^{n}} \sum_{k \in Z^{n}}\left\langle f, E_{A l} T_{B k} g^{m}(y-\cdot)\right\rangle\right|^{2} \leq C_{2}\|f\|^{2} .
$$

As a result, according to Eq. 7, Eq. 13, Eq. 16, we get

$$
C_{1}\|f\|^{2} \leq\left.\sum_{m=1}^{M} \sum_{l \in Z^{n}} \sum_{k \in Z^{n}}\left\langle f, E_{A l} T_{B k} g_{1}{ }^{m}\right\rangle\right|^{2}+\left.\sum_{m=1}^{M} \sum_{l \in Z^{n}} \sum_{k \in Z^{n}}\left\langle f, E_{A l} T_{B k} g_{2}{ }^{m}\right\rangle\right|^{2} \leq C_{2}\|f\|^{2} .
$$

Therefore, we get the proof of Theorem 3.1, which complete the proof.

Fininally, we easily get from above theorem:

Corollary. Suppose that Gabor system

$\left\{E_{A l} T_{B k} g^{m}: k \in Z^{n}, l \in Z^{n}, m=1,2, \cdots, M\right\}$

is a Parseval frame for $L^{2}\left(R^{n}\right)$, then, Gabor system

$$
\left\{E_{A l} T_{B k} g_{1}{ }^{m} \cup E_{A l} T_{B k} g_{2}{ }^{m}: k \in Z^{n}, l \in Z^{n}, m=1,2, \cdots, M\right\}
$$

is also a symmetric or antisymmetric Parseval Gabor frame about any symmetric points for $L^{2}\left(R^{n}\right)$, where the functions $g_{1}(x), g_{2}(x)$ are defined by Eq. 5 .

\section{References}

[1] R. Duffin, A. C. Schaeffer, A class of nonharmonic Fourier series, Trans. Amer. Math. Soc. 72(1952) 341-366

[2] I. Daubechies, A. Groddmann, Y. Mayer, Painless nonorthogonal expansions J. Math. Phys. 27 (1986) 1271-1283

[3] V. K. Goyal, Kovacheckeviacutec J, Kelner J A Quantized frames expansions with erasures Appl. Comput. Harmon. Anal. 10(2001) 203-233

[4] B. Hassibi, B. Hochwald, A. Shokrollahi, Representation theory for high-rate multiple-antenna code design IEEE Trans. Inform. Theory 47 (2001) 2335-2367

[5] D. Gabor, Theory of communication J. IEE (London) 93(1946) 429-457 
[6] A. Ron, Z. Shen, Weyl-Heisenberg systems and Riesz bases in $L^{2}\left(R^{d}\right)$, Duke Math. J. 89(1997) 237-282.

[7] P. G. Casazza, O. Christensen, Weyl-Heisenberg frames for subspaces of $L^{2}(R)$, Proc. Amer. Math. Soc. 129 (2001) 145-154

[8] X. L. Shi and F. Chen, Necessary conditions for Gabor frames Science in China (Series A) 50 (2007) 276-284

[9] K. Grochenig, Foundations of Time-Frequency Analysis Birkhauser Boston, MA, 2001

[10] Say Song Goh, Zhi Yuan Lim, Zuowei Shen, Symmetric and antisymmetric tight wavelet frames Appl. Comput. Harmon. Anal. 20 (2006) 3411-421

[11] Wang L F, Liu Y 2013 Symmetric Gabor frames with several generators in general lattices Int. J. Appl. Math. Stat. 50 (20) 125-131 